\title{
RAPID DETECTION OF ASHITABA (Angelica keiskei) HERBAL MEDICINE ADULTERATION USING FTIR AND PRINCIPAL COMPONENT ANALYSIS METHOD
}

\author{
Anne Yuliantini ${ }^{1, *}$, Fenti Salafiah ${ }^{1}$ and Aiyi Asnawi ${ }^{2}$ \\ ${ }^{1}$ Pharmacochemistry, Faculty of Pharmacy, Bhakti Kencana University, Jl. Soekarno Hatta No. \\ 754, Bandung 40614, West Java, Indonesia \\ ${ }^{2}$ Pharmacochemistry, School of Pharmacy, Institute Technology Bandung, Jl. Ganeca No.10, \\ Bandung 40132, West Java, Indonesia \\ *E-mail: anne.yuliantini@bku.ac.id
}

\begin{abstract}
Ashitaba is a plant used as food and herbal medicine raw material. Celery is known to be in a family and has similar leaves form with ashitaba. In this study, a rapid screening technique to detect adulteration in ashitaba herbal medicine with celery was determined using FTIR and Principal Component Analysis (PCA) method. Three samples were from a different supplier, ashitaba and celery were collected from (Indonesian region): West Java, East Java, and Riau. The samples, ashitaba, and celery leaves were extracted with maceration method using ethanol $96 \%$ and their dry extract was analyzed on FTIR with reflectant sampling handle technique. The spectral data were converted into qualitative models using Principle Component Analysis (PCA). The result of plotting PC1 and PC2 shows that ashitaba and celery separated in the different quadrant with the cumulative variance plot of PC-1 and PC-2 was $87.66 \%$ and eigenvalue at 22.06 and 11.83 . The three tested samples show that two samples suspected containing celery adulterant. The use of FTIR and PCA can potentially detect adulteration ashitaba with celery.
\end{abstract}

Keywords: Adulteration, Ashitaba, Celery, FTIR, PCA

(C) RASĀYAN. All rights reserved

\section{INTRODUCTION}

Ashitaba (Angelica keiskei) (Fig.-1a) is one of the Apiaceae family and an introduced plant originating from Japan. Ashitaba is also known as Japanese celery, angel leaf, tomorrow leaf or long-life herbal medicine. This plant is widely used as food and raw material for traditional medicine. Ashitaba has several pharmacological activities including anti-hypertriglyceridemia ${ }^{1}$, antidiabetic ${ }^{2}$, antiplatelet ${ }^{3}$, antiinflammatory, anticancer, and antioxidant.,

Celery (Apium graviolens L) (Fig.-1b) is also a member of apiaceae family which has similar morphology leaves with ashitaba. However, the content of the active compounds of these plants is different such as chalcone compound which is only owned by Ashitaba. More than 20 chalcones that are found in Ashitaba, two of are 4-hydroxyderricin and xanthoangelol which are considered to be the main active compounds for various biofunctions including anti-tumor, anti-inflammatory and anti-diabetic. ${ }^{6}$ That compounds are abundantly found in the sap and to a lesser extent in the leaf and roots $^{7}$ and to be the reasons for Ashitaba plants as potential medicinal plants. ${ }^{8}$

If celery and ashitaba are traded in the form of simplisia or powder that is almost similar to each other it will be difficult to distinguish. Besides it, Ashitaba prices are generally higher than celery in markets. This situation can be a reason for celery to be a potential adulterant of Ashitaba. If the adulteration occurs, it will certainly make customers lose. The difference of chemical compounds will be able to decrease or eliminate the biological effect produced. Therefore, it is necessary to detect adulteration in the preparation of traditional Ashitaba medicines. In this research, the detection of adulteration in ashitaba herbal medicine using FTIR spectroscopy and PCA method will be carried out.

FTIR is an instrument that uses the principle of spectroscopy which is complemented by Fourier transforms for the detection and analysis of the results of a very complex spectrum consisting of peaks

Rasayan J. Chem., 13(1), 535-540(2020)

http://dx.doi.org/10.31788/RJC.2020.1315557

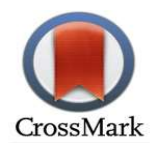




\section{RASĀYAN J. Chem. \\ Vol. 13 | No. 1 |535 - 540| January - March | 2020}

that are useful for the identification of organic compounds. ${ }^{11}$ FTIR spectroscopy has become an attractive alternative to be used as an analysis method because the required samples are small, the analysis is fast, and the use of hazardous solvents is minimized. The advantage of this method is saving time and cost. Many researchers have tried to exploit this advantage by applying FTIR to food science. Spectroscopy and chemometrics method has been used to detect adulteration, such as detecting butter adulteration with margarine ${ }^{12}$, and to authenticate of herbal medicines. ${ }^{13-15}$ The aim of this study was to detect adulteration in ashitaba herbal medicine using FTIR and PCA. This screening technique could potentially be used to analyze unknown samples for the presence of adulteration with celery.

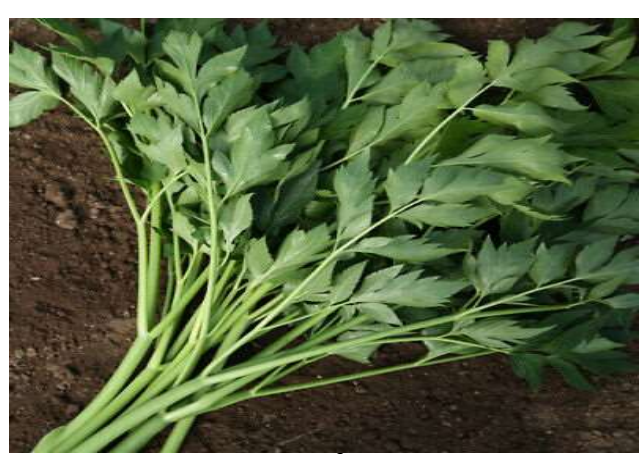

(a)

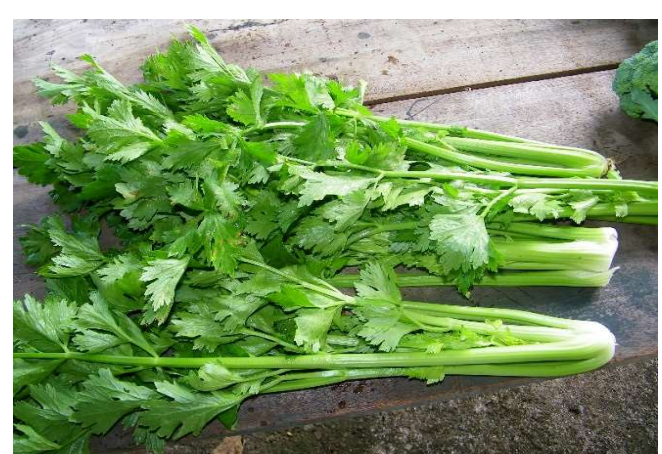

(b)

Fig.-1: (a) Ashitaba (Angelica keiskei) and (b) Celery (Apium graviolens L)

\section{Materials}

EXPERIMENTAL

Ethanol and silica gel was purchased from Merck, 3 samples ashitaba herbal medicine products were from 3 different suppliers, pipettes, beaker glasses, measuring flasks, rotary evaporator, Fourier Transform Infrared (FTIR) spectrometer (Agilent Cary 630 Type)

\section{Plant Materials}

Ashitaba (Angelica keiskei) and celery (Apium gravilens L.) leaves were collected from (Indonesia region): West Java, East Java, and Riau, 2018. The plant was identified by a botanist in the herbarium of Institute Technology Bandung of SITH, Bandung, Indonesia (Plant Identification Certificate, Number: 5974 / I1.CO2.2 / PL / 2018).

\section{Preparation of Extract}

Ashitaba and celery leaves were separated from the plant, then washed using flow water and cut into pieces. The leaves were dried by placing them in an open place with good air circulation and not exposed to direct sunlight. The dried leaves were powdered and weighed 50 grams to be extracted by maceration method using $500 \mathrm{~mL}$ ethanol solvent, soaked for 6 hours while occasionally stirring, then let stand for 24 hours. The solvent replacement was carried out 2 times with the same maceration procedure. The macerate obtained from the extraction was collected then concentrated with a rotary evaporator until viscous extract was obtained, then dried by evaporating it in a vaporizer cup with a water bath, then stored in a desiccator that contained silica gel.

\section{FTIR Spectrometry}

dried extract of Ashitaba, celery and samples were measured infrared spectrum using FT-IR spectrometer with Spectra Manager Version 2.01.03 as the application of the instrument. The FT-IR spectrum was read at a frequency of $4000-650 \mathrm{~cm}^{-1}$ with a resolution of $4 \mathrm{~cm}^{-1}$, with reflectance sample handling techniques.

\section{Data Analysis using PCA}

FTIR spectra were analyzed by the chemometric method using the Principal Component Analysis (PCA) method. PCA was done by using software R version 1.1. 463. In this study, the experiments were performed in six replicates $(n=6)$. 
RASĀYAN J. Chem.

Vol. 13 | No. 1 |535 - 540| January - March | 2020

\section{RESULTS AND DISCUSSION}

FTIR spectra of ashitaba and celery are shown in Fig.-2. The strong and distinctive spectra for ashitaba and celery were observed in the region of $650-4000 \mathrm{~cm}^{-1}$. Band one $\left(1470-1430 \mathrm{~cm}^{-1}\right)$ of celery indicate to $\mathrm{v}(\mathrm{C}-\mathrm{H})$, band two $\left(1650-1605 \mathrm{~cm}^{-1}\right)$ indicates $\mathrm{v}(\mathrm{C}=\mathrm{C})$ stretch and band three $\left(3000-2900 \mathrm{~cm}^{-1}\right)$ indicates $\mathrm{v}(\mathrm{C}-\mathrm{C})$ stretch. And in ashitaba, band one and two $\left(1065-1030 \mathrm{~cm}^{-1}\right.$ and $\left.1205-1160 \mathrm{~cm}^{-1}\right)$ belonged to $\mathrm{v}\left(\mathrm{SO}_{2}\right)$ stretch, band three $\left(1470-1415 \mathrm{~cm}^{-1}\right)$ belonged to $\mathrm{v}(\mathrm{C}-\mathrm{C})$ stretch, band four (3000$\left.2855 \mathrm{~cm}^{-1}\right)$ indicates $\mathrm{v}$ (alkyl) stretch and band five $\left(3400-3270 \mathrm{~cm}^{-1}\right)$ indicates $\mathrm{v}$ (amine).

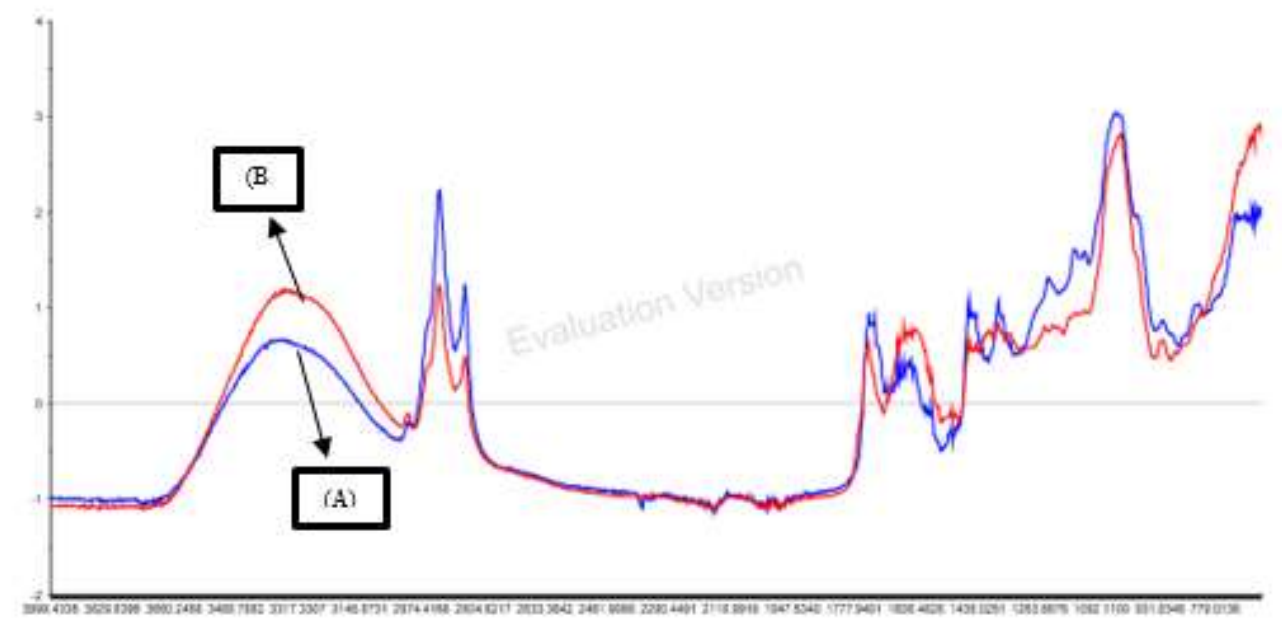

Fig.-2: FTIR Spectra of (A)Ashitaba and (B) Celery

\section{Data Analysis}

The results of FTIR spectrum measurements were further analyzed using chemometrics. This method was carried out using $\mathrm{R}$ version 1.1. 463 software. ${ }^{16}$ The chemometric method used was the Principal Component Analysis (PCA). PCA is data interpretation done by data reduction, wherein the number of matrix variables is reduced to produce new variables while maintaining the information held by the data. Validation used in PCA is cross-validation. The results of the PCA analysis are scores where each of them is obtained 3 PCs. However, the data used are only PC- 1 data against PC-2 because the grouping results are very good compared to PC-1 and PC-3. ${ }^{17}$ Based on the results of PC-1 against PC-2, a score plot curve can be made. A plot score using the first two PCs is usually the most useful because these two PCs represent the greatest variance of the data. ${ }^{18}$ The score plot curve is used to estimate the data structure as the basis for the difference between the raw extract of Ashitaba and celery extract based on geographical differences in regions. The distance between samples shows the similarity between samples. The farther the distance, the less similarity between the sample, the closer the location of the sample is to the score plot, the greater the similarity between the samples. ${ }^{19}$

The acquired data were analyzed by the application of the PCA method. The first two Principal Component (PC) were chosen to display the grouping of ashitaba as the main substance and celery as an adulterant. While the PC-1 explained $71.2 \%$ of the total variance, PC-2 explained $87.6 \%$ of the total variance. The score plot of the two PC of PC-1 versus PC-2 can be seen in Fig.-3.

And next to the FTIR spectra data of three samples were projected to PCA Score Plot of FTIR Spectra Ashitaba and celery for determining sample position whether in ashitaba or celery grouping. The result of the projection of the sample is in Fig.-4 to 6.

The result of samples projection shows that samples 1 and 3 are in celery grouping which indicates positive containing celer as an adulterant, while sample 2 is in ashitaba grouping which indicates negative containing celery as adulterant.

\section{CONCLUSION}

It can be concluded that FTIR spectroscopy in combination with PCA can be used to detect adulteration in ashitaba herbal medicine with celery as adulterant. The first two PC was used to show the grouping of 
RASĀYAN J. Chem.

Vol. 13 | No. 1 |535 - 540| January - March | 2020

ashitaba and celery with cumulative variance at $87.6 \%$. From three analyzed samples, two of them were suspected positive containing celery as an adulterant.

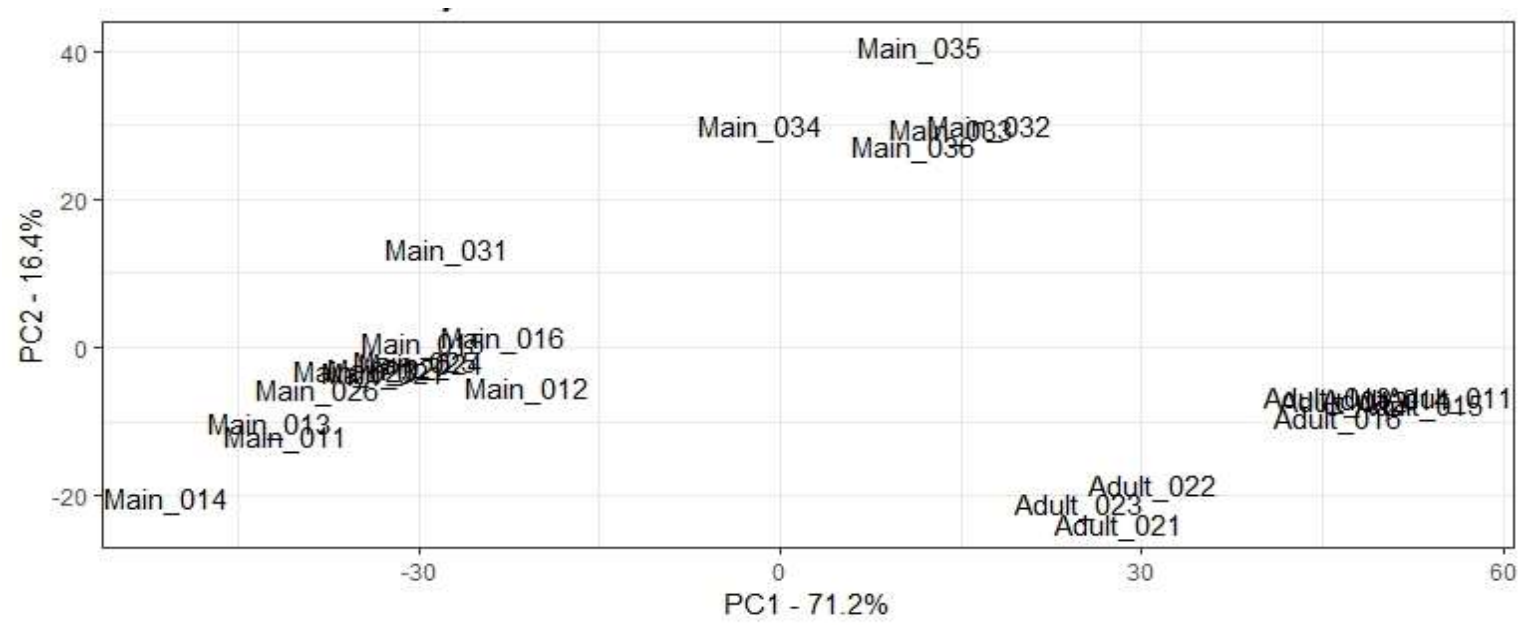

Fig.-3: PCA Score Plot of FTIR Spectra Ashitaba (Main) and Celery (Adulterant)

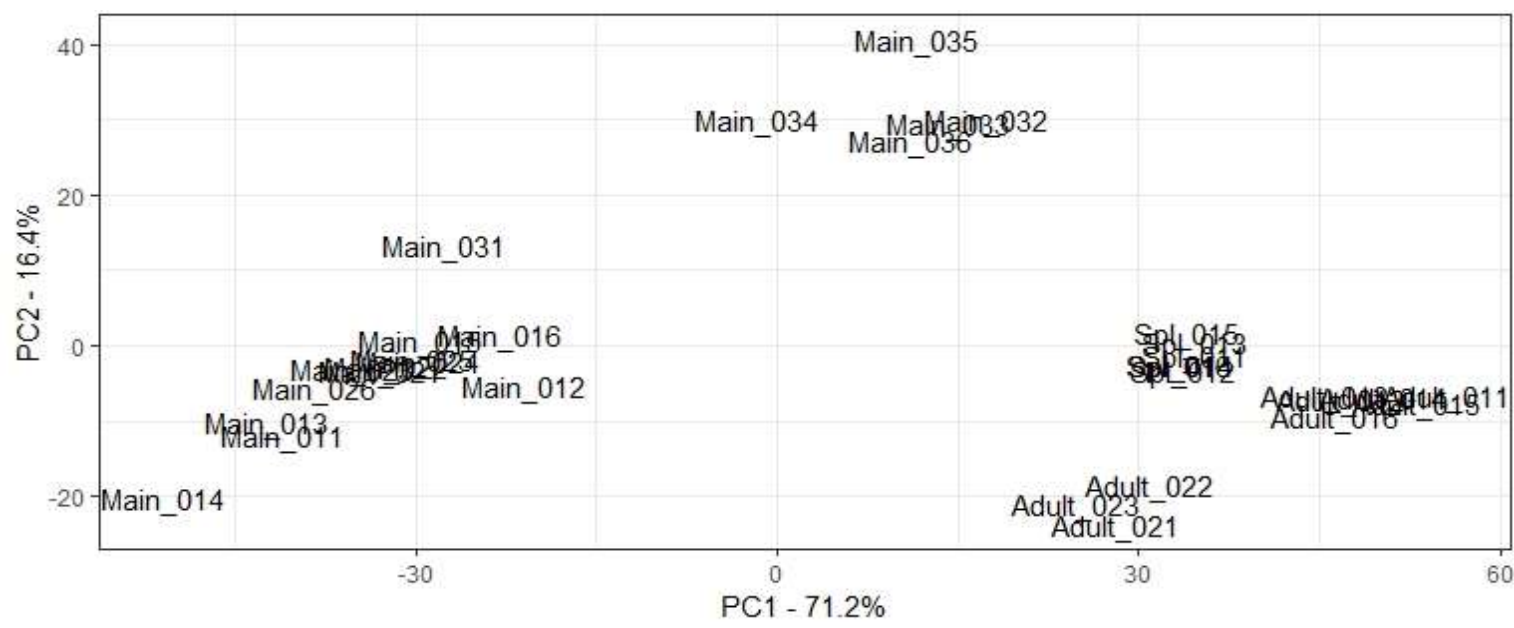

Fig.-4: Project Sample 1 onto PCA Score Plot of FTIR Spectra Ashitaba (Main) and Celery (Adulterant)



Fig.-5: Project Sample 2 onto PCA Score Plot of FTIR Spectra Ashitaba (Main) and Celery (Adulterant) 
RASĀYAN J. Chem.

Vol. 13 | No. 1 |535 - 540| January - March | 2020

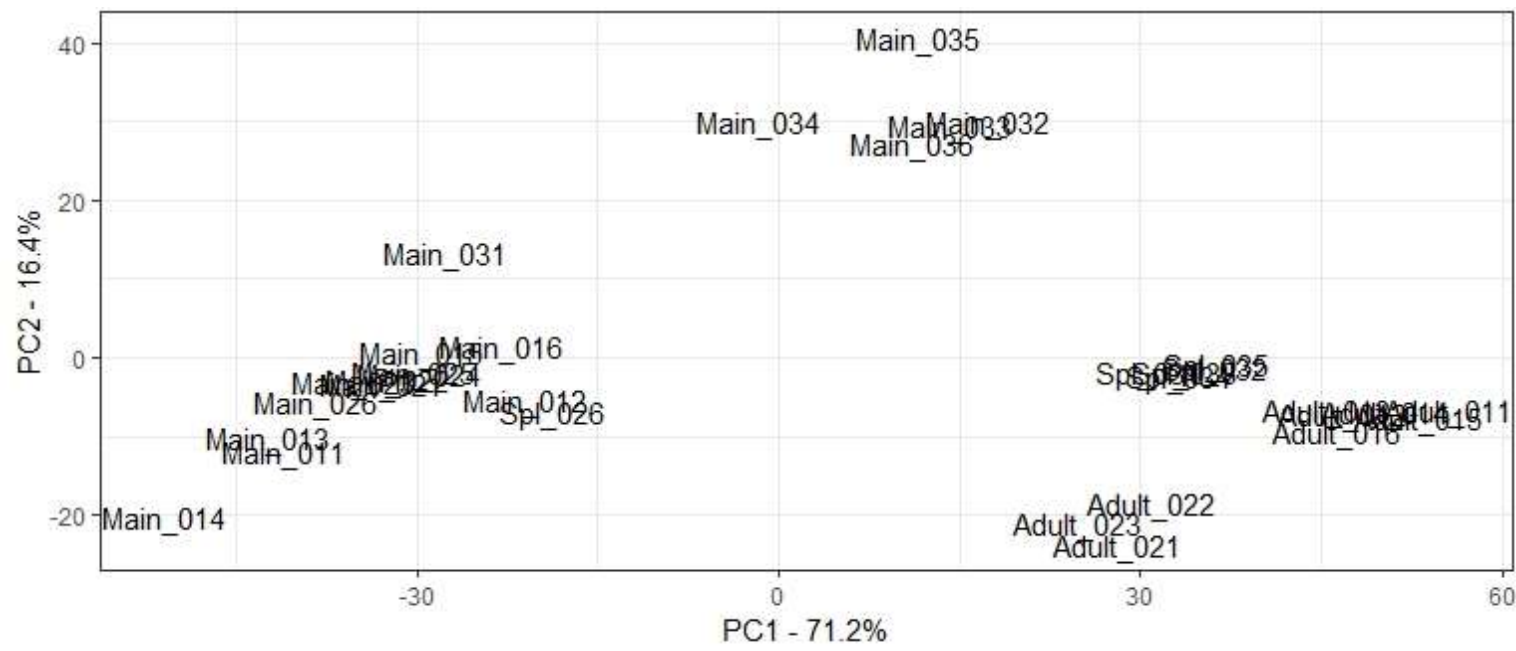

Fig.-6; Project Sample 3 onto PCA Score Plot of FTIR Spectra Ashitaba (Main) and Celery (Adulterant)

\section{ACKNOWLEDGMENT}

The author is grateful for the financial support that was provided by the research and community service institution (LPPM) Bhakti Kencana University, project number 0004/I.PE/P3M-STFB/2019

\section{REFERENCES}

1. J. Nagata, T. Morino, M. Saito, Journal of Nutritional Science and Vitaminology, 53(2), 133(2007), DOI: $10.3177 /$ jnsv. 53.133

2. T. Enoki, H. Ohnogi, K. Nagamine, Y. Kudo, K. Sugiyama, M. Tanabe, E. Kobayashi, H. Sagawa, I. Kato, Journal of Agricultural and Food Chemistry, 55(15), 6013(2007), DOI: 10.1021/jf070720q

3. D. J. Son, P. O. Park, C. Yu, S. E. Lee, Y. H. Park, Natural Product Research, 28(24), 2312(2014), DOI: $10.1080 / 14786419.2014 .931389$

4. J. R. Dimmock, D. W. Elias, M. A. Beazely, N. M. Kandepu, Current Medicinal Chemistry, 6(12), 1125(1999).

5. C. Dorn, F. Bataille, E. Gaebele, J. Heilmann, C. Hellerbrand, Food and Chemical Toxicology, 48(7), 1890(2010), DOI:10.1016/j.fct.2010.04.030

6. T. Zhang, Y. Yamashita, M. Yasuda, N. Yamamoto, H. Ashida, Food and Function, 6(1), 135(2015), DOI:10.1039/c4fo00525b

7. M. Ozawa, N. Morita, K. Baba, K. Hata, Yakugaku Zasshi, 98(5), 636(1978), DOI: 10.1248/yakushi1947.98.5_636

8. L. K. Caesar, N. B. Cech, Planta Medica, 82(14), 1236(2016), DOI: 10.1055/s-0042-110496

9. https://picclick.fr/1gabout-100pcs-Angelica-Seed-Tomorrows-Leaf-Ashitaba-Angelica113394301931.html

10. https://www.wellgrowseeds.com/product/apium-graveolens-secalinum-leaf-celery-westland-1gmapprox-2500-seeds/

11. A. Subramanian, V. B. Alvarez, W. J. Harper, L. E. Rodriguez-Saona, International Dairy Journal, 21, 434(2011), DOI:10.1016/j.idairyj.2010.12.012

12. R. S. Uysal, I. H. Boyaci, H. E. Genis, U. Tamer, Food Chemistry, 141, 4397(2013), DOI: 10.1016/j.foodchem.2013.06.061

13. H. A. Gad, S. H. El-Ahmady, M. I. Abou-Shoer, M. M. Al-Azizi, Phytochemical Analysis, 24, 1(2013), DOI: 10.1002/pca.2378

14. S. Sun, J. Chen, Q. Zhou, G. Lu, K. Chan, Planta Medica, 76, 1987(2010), DOI:10.1055/s-00301250520

15. H. B. Zou, G. S. Yang, Z. R. Qin, W. Q. Jiang, A. Q. Du, H. Y. Aboul-Enein, Analytical Letters, 38, 1457(2005), DOI:10.1081/AL-200062153 
RASĀYAN J. Chem.

Vol. 13 | No. 1 |535 - 540| January - March | 2020

16. R. Team, RStudio: Integrated development for R (Version 1.1. 463)[Computer Software], (2018).

17. R. C. Team, R: A Language and Environment for Statistical Computing, (2013), DOI: 10.1890/00129658(2002)083[3097:CFHIWS]2.0.CO;2

18. Wickham, ggplot2: Elegant Graphics for Data Analysis, Springer, (2016), DOI: 10.1007/978 -0-38798141-3

19. Husson, S. Lê, J. Pagès, Exploratory Multivariate Analysis by Example using R. Chapman and Hall/CRC (2018), DOI: 10.1201/b21874

[RJC-5557/2019] 